\title{
Heterogeneity and Environmental Preferences Shape the Evolution of Cooperation in Supply Networks
}

\author{
Dong Mu $(\mathbb{D})$ and Xiongping Yue \\ School of Economics and Management, Beijing Jiaotong University, Beijing 100044, China \\ Correspondence should be addressed to Xiongping Yue; 18113043@bjtu.edu.cn
}

Received 1 September 2020; Revised 21 March 2021; Accepted 2 April 2021; Published 20 April 2021

Academic Editor: Danilo Comminiello

Copyright (C) 2021 Dong Mu and Xiongping Yue. This is an open access article distributed under the Creative Commons Attribution License, which permits unrestricted use, distribution, and reproduction in any medium, provided the original work is properly cited.

\begin{abstract}
Supply networks as complex systems are significant challenges for decision-makers in predicting the evolution of cooperation among firms. The impact of environmental heterogeneity on firms is critical. Environment-based preference selection plays a pivotal role in clarifying the existence and maintenance of cooperation in supply networks. This paper explores the implication of the heterogeneity of environment and environment-based preference on the evolution of cooperation in supply networks. Cellular automata are considered to examine the synchronized evolution of cooperation and defection across supply networks. The Prisoner's Dilemma Game and Snowdrift Game reward schemes have been formed, and the heterogeneous environment and environmental preference have been applied. The results show that the heterogeneous environment's degree leads to higher cooperation for both Prisoner's Dilemma Game and Snowdrift Game. We also probe into the impact of the environmental preference on the evolution of cooperation, and the results of which confirm the usefulness of preference of environment. This work offers a valuable perspective to improve the level of cooperation among firms and understand the evolution of cooperation in supply networks.
\end{abstract}

\section{Introduction}

Supply networks are composed of numerous firms from buyers and suppliers, and links are supply relationships formed by exchanging goods and services [1]. Within the scope of buyer-supplier relationships, the supply network needs consideration of many firms from broadening dimensions and factors. The complexity of the network is difficult for decision-making in predicting the consequence of the strategic decisions of firms $[2,3]$. Changes in a single firm strategy will influence the emerging properties of the entire network [4]. Large-scale supply networks are complex systems and not supported by simple hierarchical models $[5,6]$. In a dynamic environment, the supply network is constantly shifting strategies and objectives, and the emergent properties of cooperation among firms are difficult to understand and explore.

In the network's cooperation strategy, the sanction is destructive for cooperative behaviour in a supply chain.
Structural embedding provides an environment that facilitates adaptive collaborative behaviour for firms that are part of the supply network [7]. Adaptive and probabilistic strategies are studied to explore the universality of cooperation in Prisoner's Dilemma $[8,9]$. In the cooperative game, the Nash bargaining solution concept is used to study the collision behaviour of suppliers [10]. Larger groups are less cooperative in the N-person Prisoner's Dilemma but more cooperative in the Public Goods game [11]. More massive clusters have more substantial resource concentration capabilities. At the same time, firms with more resources tend to work more together [12]. The higher incomes earned may justify the risk intrinsic in cooperation, particularly for uncertain firms [13]. As long as the game revenue-cost ratio is higher than network connectivity, reciprocity and multistep prediction horizon are necessary for stable collaboration and sufficient for fixed cooperation [14]. The facilitation of cooperative behaviour mainly depends on participants' weight distribution, which is based on 
the formation of cooperative clusters controlled by highweight collaborators [15]. The system's general description is derived using ordinary differential equations, which provides a common framework to simulate and quantify the effects of single-node dynamics on the macroscopic state of the network [16]. The more survivable a network is, the more dependable it will be [17]. The stronger the level of asymmetry, the higher the level of cooperation [18]. Sakiyama and Arizono find that a novel spatial Hawk-Dove model generates a feature population pattern and represents the collaborators' survival, where the updated rules are fixed compared to the classical spatial Hawk-Dove model [19]. Comparing three typical rules, such as unconditional imitation, replicator dynamics, and the Moran process, finds that the Moran process can mainly improve the frequency of cooperation [20]. Although firms always pursue profit maximization, the inherent differences in member interests make it difficult to achieve full cooperation. Therefore, it is significant to explore the evolution of cooperation in the context of supply networks.

Heterogeneity has been investigated to promote cooperation in the recent literature [21-24]. Heterogeneous networks are more conducive to cooperative behaviour than homogeneous networks [25]. Individual heterogeneity confirms the existing evidence that heterogeneity promotes cooperative behaviour almost regardless of its origin [26]. Colon and Ghil have demonstrated that the heterogeneity of the delay value significantly affects the economic network's response to small, localized perturbations [27]. Firms in collaborative networks are characterized by being heterogeneous and autonomous. Andres and Poler propose a DDS for the collaborative selection of consistent strategies between firms belonging to the collaborative networks [28,29]. Heterogeneity in product quality decay leads to different logistics network structure [30]. As the heterogeneity of supplier base costs increases, the optimal number of suppliers required decreases [31]. Valuation heterogeneity between manufacturers can mitigate unfavourable supply and demand balances, thereby protecting some of the manufacturer's surplus and leading to higher price dispersion in the supply chain network [31]. The problem of spectrum allocation in cognitive radio networks based on combined auctions is studied while considering spectrum supply heterogeneity and demand [32]. Liu investigated the interplay between awareness and risk propagation in $\mathrm{R} \& \mathrm{D}$ networks considering firms' heterogeneity [33].

Preference selection has received more attention for the evolution of cooperation in networks $[34,35]$. Firms have a strong preference for geographic locations suitable for multimodal and multimodal transport [34]. The investment preference among firms can give rise to cooperation [35]. The firm's strategy preference can affect others' strategy selection, and the density of cooperation presents a growth within a wide range of value of strategy preference [36]. The level of cooperation decreases with an increase in the degree of risk preference [37]. The ambiguity of risk preference and potential probability distribution has been incorporated into a robust supply chain network design [38]. Increasing the level of preference can promote the behaviour of cooperation [39]. Firms like to choose neighbours with a significant degree of difference to learn, regardless of the network structure, and there is the highest preference intensity leading to the most significant level of cooperation [40].

Inspired by their work in supply networks, all of the firms are different. Firms are surrounded by neighbours with different properties, indicating that firms are in a heterogeneous environment. On the other hand, the firms' fitness is derived not only from interactions with neighbours but also in terms of the payoff of environmental heterogeneity in the supply network. Therefore, we incorporate the heterogeneity of the environment into the fitness of firms. The heterogeneous environment signifies that when the focal firm's payoff is higher than the supplier's, it will increase the focal firm's fitness, for the focal firm is a more influential leader in the supply networks, which usually obtain additional payoff $[41,42]$. On the contrary, when the focal firm's payoff is less than the supplier's income, it will decrease. If the supplier's payoff is larger than the focal firm's, which surrounded by high-efficiency firms, the focal firm will soon bankrupt due to overcrowding and difficulty obtaining payoff. There is no doubt that preference would affect the firm's decision during strategy updating [36]. Firms can take response according to their strategy and other strategies. In supply networks, firms are more inclined to adopt a strategy with higher fitness. The preference of the environment means that the suppliers who have higher fitness are more likely to be selected by the focal firm to adapt its strategy. The evolutionary Prisoner's Dilemma Game (PDG) and Snowdrift Game (SDG) are employed to explore the impact of heterogeneity and preference of environment on the evolution of cooperation in supply networks.

The purpose of our research is to investigate the implication of the heterogeneity and preference of environment on an evolving supply network and address the following questions:

(1) How does the heterogeneity of the environment in the supply network affect the evolution of cooperation in the two major types of games between firms?

(2) How does the preference of environment in the supply network affect the evolution of cooperation in the two major types of games between firms?

(3) How does the heterogeneity and preference of environment in the supply network affect the evolution of cooperation in the two major types of games between firms?

In this paper, we use a cellular automata (CA) simulation framework to model firms' interaction within the supply networks. Simulations have been established to answer research questions using the PDG and SDG rewards schemes, representing various payoff of interaction. The experimental results show that the coevolution of firms' strategies produces interesting properties of emergency.

This paper is organized as follows: Section 2 describes some settings of methodology. We present the simulation results and analysis in Section 3. After this, discussion and conclusions are given in Section 4. 


\section{Methodology}

The methodology was developed following the process, as shown in the flowchart in Figure 1.

From Figure 1, we can see that six sequential steps of the modelling steps, for simplicity, turn six steps into three main parts. Firstly, supply networks were established, which described the structure of numerous firms. Secondly, two major types of games were presented: firms play the game with their nearest neighbours and obtain their payoff. Finally, the heterogeneous environment and the preference of the environment were designed. The three main parts are introduced in this section.

For convenience, we here list some symbols in Table 1.

2.1. Supply Network Context. A supply network consists of numerous interacted firms engaged in suppliers, manufactures, or retailers $[43,44]$. We observe that the buying company is permanently embedded in the supply network and is associated with the supplier company that forms the basis of its supply [45-47]. In the context of the CA model, the firms are expressed as a cell. For two-dimensional CA, there are two significant types founded: the Moore neighbourhood, comprising the eight adjacent cells; the Neumann neighbourhood, incorporating the four adjacent cells. We proposed that buyers as focal cells and focal firms would have eight neighbouring (the Moore neighbourhood) firms that form a supply network [48]. Firms interact with their nearest eight neighbours which interact with buyers, and a firm's change will influence the entire supply network, as depicted in Figure 2. We can see that a firm acts as a buyer (focal firm) in one interfirm whereas the same firm acts as a supplier $(\mathrm{N})$ in another interaction. Therefore, it is necessary to figure out the implication of firms' decisions in the light of reciprocity.

In the CA model, firms have cooperation and defection strategies, a synchronized evolution of strategies across the network [48]. The firms will have a strategy in the initial state, cooperation, or defection. In each step, the firms and the nearest eight neighbours play a game to obtain payoff. According to the payoff gained, the strategy chosen by the company for the next step is determined. By evaluating simulation behaviour, we can gather insights about the evolution of cooperation and defection in the supply network.

2.2. Game Reward Schemes. The PDG and SDG are diffusely applicable games to investigate the evolution of cooperation [49]. Two players engage in a round of the game. The player will get a payoff $R$ if they both choose cooperation and receive a payoff $P$ if they both choose defection. If one player cooperates but the other defects, the cooperator will accept payoff $S$, and the defector's payoff is $T$ and game reward schemes are used, as shown in Table 1 . We have the weak PDG $[39,50]$, if $T>R>P>S$ and $2 R>T+S$, and the SDG if $T>R>S>P$ [51], and this covers two major types of social dilemmas that players can choose between cooperation and

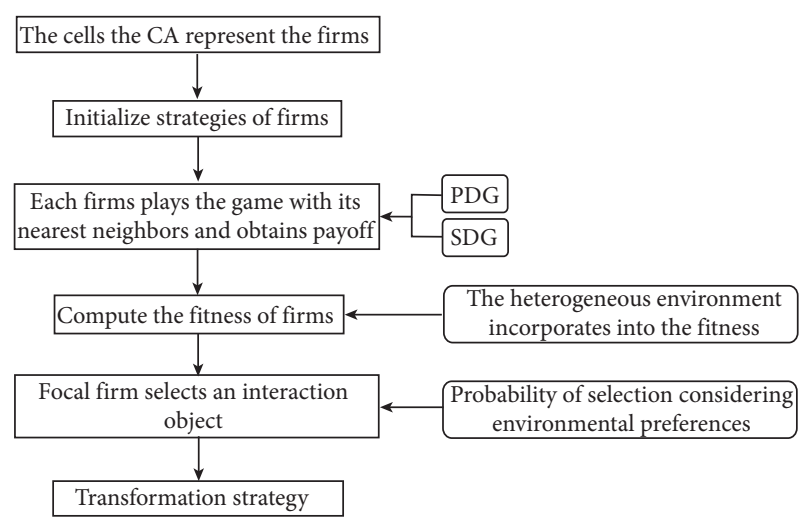

FIGURE 1: Flowchart of the methodology.

defection. For simplicity $[52,53]$, but without loss generality, the parameters in PDG are as follows: $R=1, P=S=0$, and $T=b(1 \leq b \leq 2)$, as shown in Table 2; the parameters in SDG are as follows: $[R=1, P=0, T=1+r$, and $S=1-r$, as shown in Table 3.

At the beginning of the game, each player randomly chooses strategies within cooperation and defection. At each step, each player plays the game with its nearest neighbours and obtains its payoff, and the accumulated payoff of player $i$ can be signified as $P_{i}$ :

$$
P_{i}=\sum_{j \in N_{i}} S_{i}^{T} M_{T_{i}} S_{j},
$$

where $N_{i}$ indicate the number of nearest neighbours of player $i . S_{i}$ means that the player $i$ can choose one of the strategies: cooperation or defection, $S_{i}=(1,0)^{T}$ or $(0,1)^{T}$. $M_{T_{i}}$ represents the matrix payoff of the game.

2.3. The Heterogeneity and Preference of Environment. We assume that the player's fitness is also affected by the nearest neighbour's level of the environment. The environment of the player $i$ is expressed by the average of its nearest neighbours' payoff $P_{j}$, and we can compute the degree of heterogeneity of the environment in the following formulation $[50,54]$ :

$$
\overline{P_{i}}=\frac{\sum_{j=1}^{N_{i}} P_{j}}{N_{i}} .
$$

We evaluate the fitness of the player $i$ as follows:

$$
F_{i}=P_{i}+u *\left(P_{i}-\bar{P}_{i}\right),
$$

where the tunable parameter $u \in[0,1]$ signifies the contribution of the environment to fitness, when $u=0$ implies that the environment has no effect on fitness, and $F_{i}$ equals $P_{i}$, where the player fitness is homogeneous. If $u \neq 0$ means that the environment incorporates into the fitness, furthermore, the environment increases (decreases) the fitness when $P_{i}>\bar{P}_{i}\left(P_{i}<\bar{P}_{i}\right)$.

Then, the player $i$ will select an interaction object $j$ with the following probability [39]: 
TABLE 1: Some symbols and the corresponding meanings.

\begin{tabular}{lr}
\hline Notation & Meaning \\
\hline$R$ & The payoff if they both choose cooperation \\
$S$ & The payoff of the defector, if one player cooperates but the other defects \\
$P$ & $\begin{array}{c}\text { The payoff of cooperator, if one player cooperates but the other defects } \\
\text { The payoff if they both choose defection } \\
N_{i}\end{array}$ \\
$S_{i}$ & $\begin{array}{c}\text { The number of nearest neighbours of the player } i \\
M_{T_{i}}\end{array}$ \\
$P_{i}$ & $\begin{array}{c}\text { The player } i \text { can choose one of the strategies: cooperation or defection } \\
P_{i}\end{array}$ \\
$F_{i}$ & The matrix payoff of the game \\
$u$ & The accumulated payoff of player $i$ \\
$\alpha$ & The degree of the heterogeneous environment \\
$b$ & The fitness of the player $i$ \\
$r$ & The degree of contribution of the environment to the fitness \\
The degree of preference for the environmental level & The temptation to defect \\
The cost-to-benefit rate
\end{tabular}

\begin{tabular}{|l|c|c|c|l|}
\hline$N_{11}$ & $N_{18}$ & $N_{17}$ & & \\
\hline$N_{12}$ & $\begin{array}{c}N_{1} \\
\text { (Focal firm) }\end{array}$ & $\begin{array}{c}N_{8} \\
\left(\mathrm{~N}_{16}\right)\end{array}$ & $N_{7}$ & \\
\hline$N_{13}$ & $\begin{array}{c}N_{2} \\
\left(N_{14}\right)\end{array}$ & $\begin{array}{c}\text { Focal firm } \\
\left(N_{15}\right)\end{array}$ & $N_{6}$ & \\
\hline & $N_{3}$ & $N_{4}$ & $N_{5}$ & \\
\hline & & & & \\
\hline
\end{tabular}

Figure 2: The changing role of the focal firm (buyer) and supplier in supply networks.

TABLe 2: PDG reward scheme.

\begin{tabular}{cccc}
\hline & & \multicolumn{2}{c}{ Player 2} \\
& & Cooperation & Defection \\
\hline \multirow{2}{*}{ Player 1} & Cooperation & 1,1 & $0, b$ \\
& Defection & $b, 0$ & 0,0 \\
\hline
\end{tabular}

TABLE 3: SDG reward scheme.

\begin{tabular}{lccc}
\hline & & \multicolumn{2}{c}{ Player 2} \\
& & Cooperation & Defection \\
\hline \multirow{2}{*}{ Player 1} & Cooperation & 1,1 & $1-r, 1+r$ \\
& Defection & $1+r, 1-r$ & 0,0 \\
\hline
\end{tabular}

$$
\pi_{j}=\frac{\exp \left(\alpha * P_{j}\right)}{\sum_{j=1}^{N_{i}} \exp \left(\alpha * P_{j}\right)},
$$

where $\alpha$ indicates the degree of preference for the environmental level, and the range goes from 0 to 1 . When $\alpha=0$, the model will turn to the traditional occasion where an interaction object is randomly selected from neighbours in the supply network. At the same time, when $\alpha>0$, the environmental preference of players is presented [55], and the focal player prefers to adopt the strategy which has a higher level of the environment.

In every time step, the firms in supply networks interact with each other and, at the end of the time step, update their strategy to cooperate or to defect in the next time step based on the strategy that resulted in the highest fitness. If $F_{i} \geq F_{j}$, the player $i$ keeps the strategy $S_{i}$; if $F_{i}<F_{j}$, it updates $S_{i}$ into $S_{j}$ [48]. Through an evaluation of the simulated behaviours over time, we can glean insights regarding the evolution of cooperation and defection in supply networks.

\section{Results}

A series of simulations have been conducted for understanding the evolution of cooperation. The first simulation concentrates on the implications of the heterogeneity of the environment, and an extended simulation was designed to investigate the correlation between environmental preference and the density of cooperation. The third simulation was used to figure out the influences of the coexistence of environmental heterogeneity and preference.

3.1. Implications of the Heterogeneity of the Environment. We first examine the impact of heterogeneity of the environment on the emerging and sustainable properties of cooperation in PDG. For the time evolution of cooperation for different values of $u$, furthermore, we have used $\alpha=0$ and $b=1.3$, and the result is shown in Figure 3. The resulting network reciprocity should be considered to have two main aspects, END and EXP, which have been defined in previous 


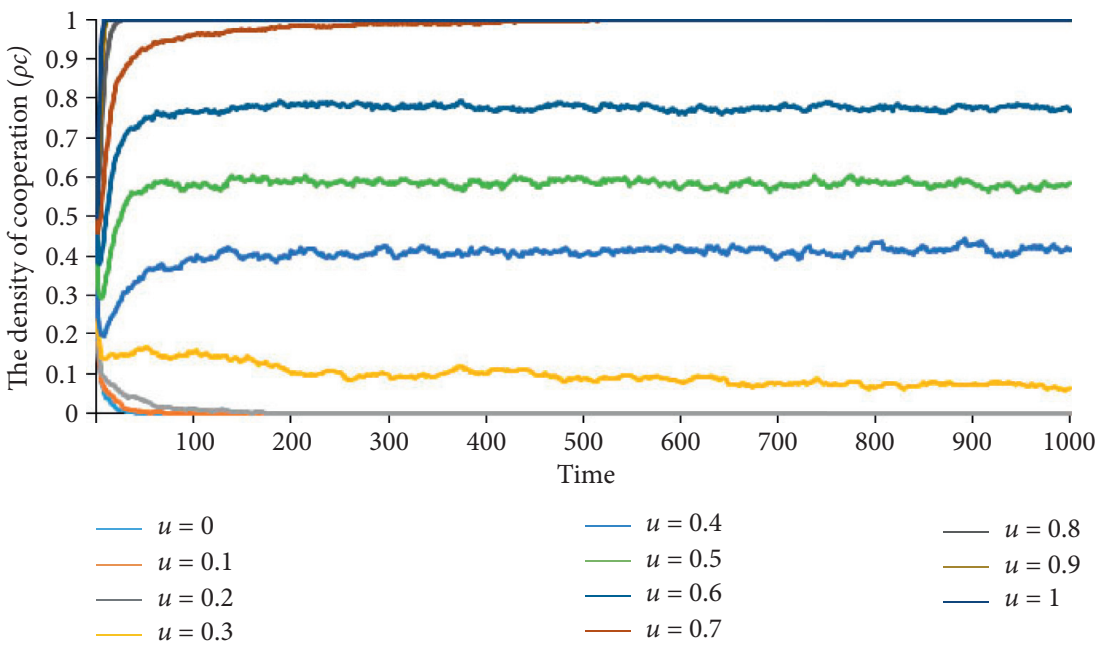

Figure 3: Time course of the density of cooperation for different values of $u$, the depicted is obtained in PDG for $\alpha=0$ and $b=1.3$. It is observed that the density of cooperation will rise with the increase of $u$.

studies [56, 57]. END refers to when the global cooperation faction, which started an evolutionary path, decreases from the initial arrangement of cooperators and defectors. EXP refers to a period when the global cooperation fraction increases. The evolutionary path is absorbed by an all-defectors state in the END, when $u=0,0.1$, and 0.2 , and 0.3 . The density of cooperation will rise with the increase of $u$, and three different types are founded: only defection, mix-strategies, and only cooperation. When $u=0,0.1$, or 0.2 , all firms choose defection, and cooperators will soon die. Especially, for $u=0$, the model turns into a traditional version. It should be noticed that the time steps to attain complete defection is a significant difference. The minor heterogeneity of the environment parameter $u$, the less time it will use. The cooperator hardly survives to keep the cooperation strategy for $u=0.3$ or 0.4 , and defective behaviour dominates in the supply network. The cooperators interact with cooperative neighbours can withstand the defector's invasion, and the strategy of cooperation dominates in the supply network when $u=0.5$ or 0.6 . The parameter value $u$ is $0.7,0.8,0.9$, or 1 , and all firms choose to cooperate. The bigger parameter $u$, the less time it will take to achieve full cooperation.

We have investigated the implication of the environment's heterogeneity on the density of cooperation in SDG, as shown in Figure 4. For the SDG, the density of cooperation will rise with the increase of $u$. Similar to the outcome of the PDG, three types are founded: only cooperation, mix-strategies, and only defection. The evolutionary path is absorbed by an all-defector state in the EXP when $u=0.8$, 0.9 , and 1 . All firms choose to cooperate rapidly when $u=0$ or 0.1 , but the time step to attaining complete cooperation is a noticeable difference. The minor heterogeneity of the environment $u$, the less time it will take. Especially, for $u=0$, we cannot consider the heterogeneity of the environment mechanism that leads to the model transit into a traditional SDG. With the increase of the environment's heterogeneity $u$, the strategy of firms tends to cooperate, but defective behaviour will also dominate when $u=0.2,0.3,0.4,0.5,0.6$, or 0.7. Cooperative behaviour dominates when $r=0.8$. In both cooperation dominates and defection dominates cases, the number of cooperating firms fluctuates less and is almost stable at a specific value. All firms will choose to cooperate when $u=0.9$, or 1 .

We have investigated how cooperation evolves when either the parameter $u$ or the parameter $\alpha$ is fixed at 0 . In order to demonstrate the impact of the temptation to defect $b$ and the heterogeneity of the environment $u$ on the evolution of cooperation in PDG, the parameter $\alpha$ is fixed at 2, and the result is depicted in Figure 5. When $\alpha=2$, the firms have an environmental preference. All the firms prefer to adopt the strategy, which has a higher level of the environment. Two significant results can be found: first of all, the $u-\alpha$ parameter plane can be divided into three types, where the cooperation level is roughly the same. Most of the area is filled with yellow and light-yellow colour, which represents a high density of cooperation. Moreover, as the value of $u$ becomes more significant, the density of cooperation gradually expands. For example, when $b=2$, the colour gradually changes from blue to yellow with the increase of $u$. Especially, for $u>0.8$, regardless of the value of $b$, the density of cooperation is 1 .

In order to demonstrate the impact of the cost-to-benefit $r$ and the heterogeneity of the environment $u$ on the evolution of cooperation in SDG, the parameter $\alpha$ is fixed at 2, and the result is shown in Figure 6. We can see that the complete defection occupied the smaller proportion of the plane, which expresses that cooperation dominates. When $u=0$, the firm's fitness is merely depending on the game payoff, and the environmental preference mechanism is considered. The density of cooperation is 0 for $r=0.9$. With the increment of $u$, the lager density of cooperation. Especially, for $u>0.8$, all firms will choose to cooperate regardless of the value of $r$. Increasing the value of $u$ enhances the degree of the heterogeneous environment, which plays a significant role in advancing cooperation.

3.2. Implications of the Preference of Environment. To investigate the implication of the environment preference mechanism on the evolution of cooperation in PDG, the 


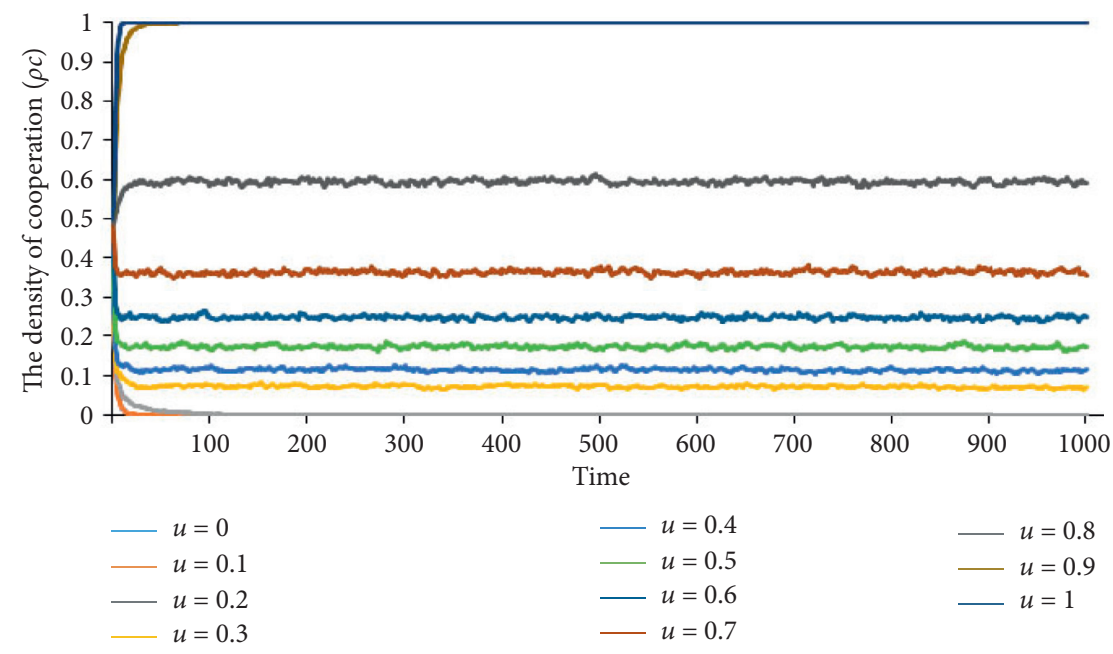

FIgUre 4: Time course of the density of cooperation for different values of $u$, the depicted is obtained in SDG for $\alpha=0$ and $r=0.8$. It is observed that the density of cooperation will rise with the increase of $u$.

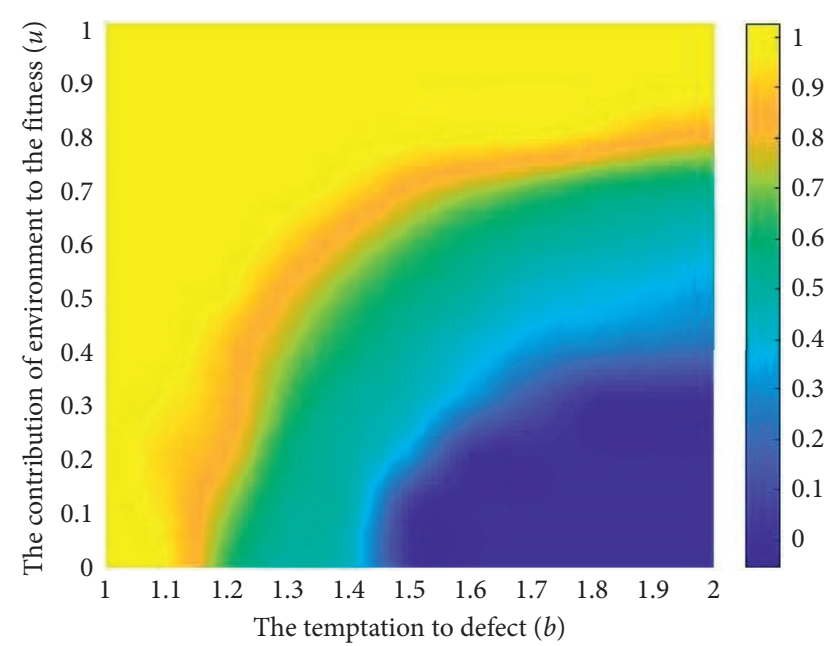

Figure 5: The colour-coded (see the bar on the right) density of cooperation $\rho_{c}$ on the $u-b$ parameter plane. The depicted are obtained in PDG for $\alpha=2$. The yellow shading corresponds to the higher stationary density of cooperators at each particular combination of $u$ and $b$. It can be observed that $u$ promotes cooperation better than the $b$.

value of $\alpha$ is varied from 0 to 5 with an interval of 1 . We have used $u=0$ and $b=1.3$, and the result is shown in Figure 7 . Especially, when $\alpha=0$, the model turns into the traditional type in which an interaction object is randomly selected from neighbours. The evolutionary path is absorbed by an all-defector state in the END, when $\alpha=0$ and 1 . However, the evolutionary path is absorbed by an all-defector state in the EXP, when $\alpha=2,3$, and 4 , and 5 . Three remarkable results can be found: first, the environmental preference mechanism between firms improves the cooperation strategy in supply networks. It is observed from the figure that the cooperators soon disappear in the traditional version. However, the density of cooperative firms increased compared to the traditional version when $\alpha>0$. The more considerable value of $\alpha$, the higher level of density of

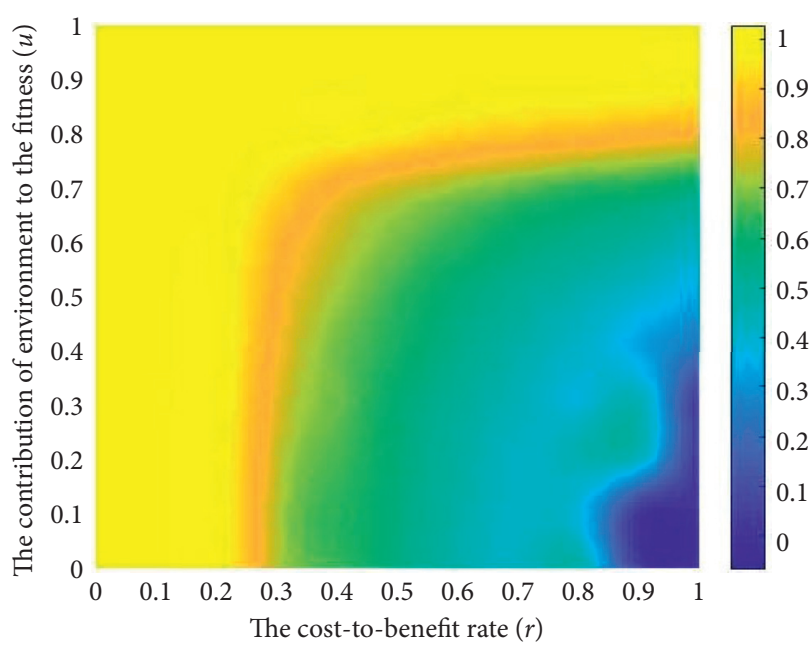

FIGURE 6: The colour-coded (see the bar on the right) density of cooperation $\rho_{c}$ on the $u-r$ parameter plane. The depicted are obtained in SDG for $\alpha=2$. The yellow shading corresponds to the higher stationary density of cooperators at each particular combination of $u$ and $r$. It can be observed that $u$ promotes cooperation better than the $r$.

cooperation. Second, there are differences in the magnitude of growth of density. The density of cooperation increases the most when $\alpha$ is 1 to 2 . Third, the density of cooperating firms gradually increases and finally stabilizes at a particular value when $\alpha=2,3,4$, or 5 , and it decreases when $\alpha=0$ or 1 .

In order to demonstrate the impact of the environmental preference on the evolution of cooperation in SDG, the parameter $u$ is fixed at 0 and $r=0.8$, and the result is shown in Figure 8 . The evolutionary path is absorbed by an alldefector state in the END, when $\alpha=0$ and 1 . However, the evolutionary path is absorbed by an all-defector state in the EXP, when $\alpha=2,3$, and 4 , and 5 . Two relevant results can be found: first, the environmental preference can promote the density of cooperation. It is observed from the figure that the cooperators soon disappear in the traditional version. 


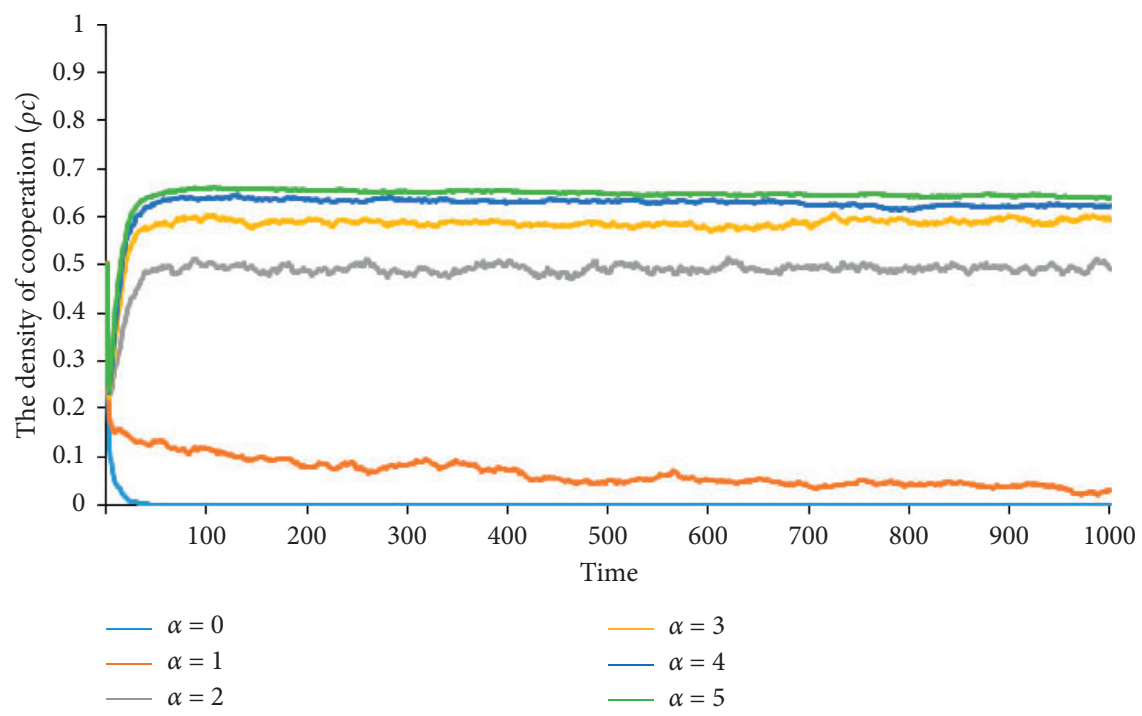

FIGURE 7: Time course of the density of cooperation for different values of $\alpha$, the depicted is obtained in PDG for $u=0$ and $b=1.3$. It is observed that the density of cooperation will rise with the increase of $\alpha$.

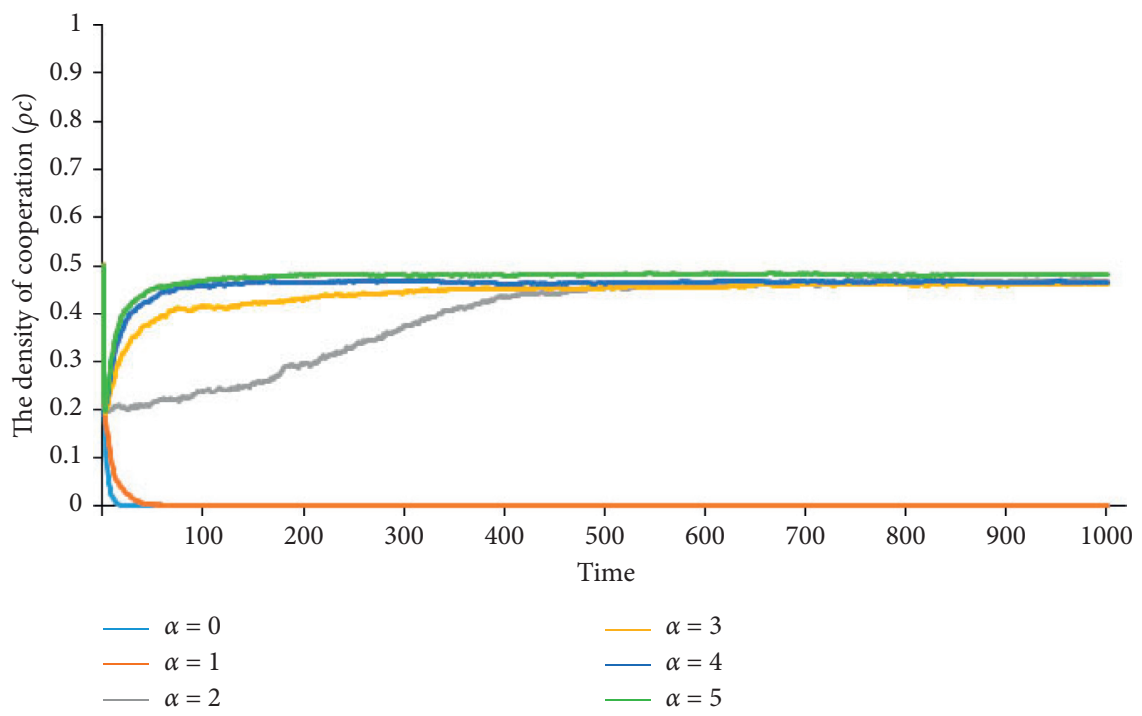

FIGURE 8: Time course of the density of cooperation for different values of $\alpha$, the depicted is obtained in SDG for $u=0$ and $r=0.8$. It is observed that the density of cooperation will rise with the increase of $\alpha$.

However, the density of cooperation increased to 0.5 when $\alpha=2,3,4$, or 5 . Second, the density of cooperating firms gradually increases and finally stabilizes at a specific value. The time steps to attain a particular value are significant. The broader the environmental preference parameter $\alpha$, the less time it will use.

To thoroughly investigate the influences of the temptation to defect $b$ and the environmental mechanism on the evolution of cooperation, Figure 9, in which the parameter $u=0.2$, indicates how $\rho_{c}$ varies in dependence on the temptation to defect $b$ for different values of $\alpha$. The whole plane is divided into three types: complete defection, mixstrategies, and full cooperation. When $\alpha=0$, the model turns into PDG, which the strategy of cooperation based on the temptation to defect $b$ and $u=0.2$, and the firms are entirely choosing to defect when $b>1.3$. The density of cooperators, shown by the blue bar, varies around 0 when $b=2$. For larger values of $\alpha$, we can see that the environmental mechanism has an impact on the density of cooperation, and some planes are yellow and green colour, which represents a high density of cooperators.

Figure 10 , in which the parameter $u=0.2$, indicates how $\rho_{c}$ varies in dependence on the temptation to defect $r$ for different values of $\alpha$. The whole plane is also divided into three types: full defection, mix-strategies, and full cooperation. It is worth noting that the full cooperation occupied the one-third proportion of plane, which expresses that cooperation is promoted by more significant $\alpha$. The large proportion of the mix-strategies plane is presented in Figure 4. In particular, defection dominates when $r=1$ 


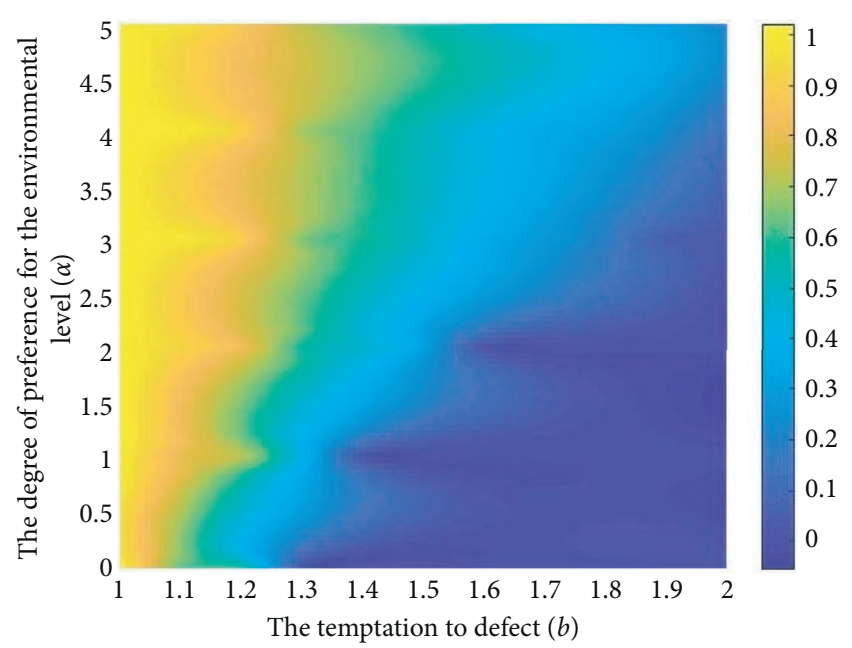

FIgURE 9: The colour-coded (see the bar on the right) density of cooperation $\rho_{c}$ on the $\alpha-b$ parameter plane. The depicted are obtained in the PDG for $u=0.2$. The yellow shading corresponds to the higher stationary density of cooperators at each particular combination of $\alpha$ and $b$.

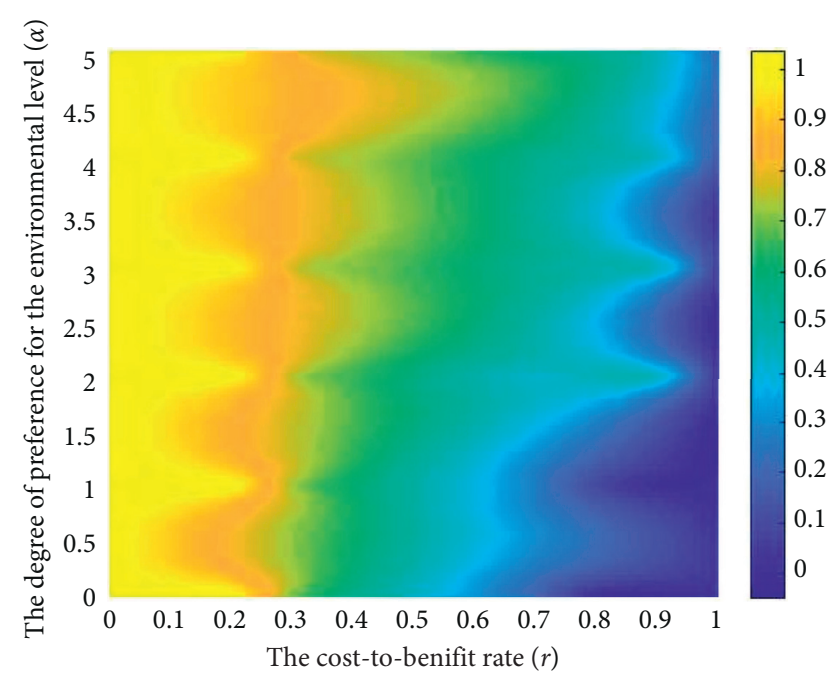

FIGURE 10: The colour-coded (see the bar on the right) density of cooperation $\rho_{c}$ on the $\alpha-r$ parameter plane. The depicted are obtained in the SDG for $u=0.2$. The yellow shading corresponds to the higher stationary density of cooperators at each particular combination of $\alpha$ and $r$.

regardless of the value of $\alpha$. In general, the environmental preference mechanism can guarantee a beneficial environment for cooperation.

\subsection{Implications of the Heterogeneity and Preference of} Environment. We describe the implications of the heterogeneity and preference of the environment on the density of cooperation. In PDG, the temptation to defect $b=1.3$, as depicted in Figure 11(a). In SDG, the cost-to-benefit rate $r=0.8$, as depicted in Figure 11(b). When $\alpha=0$ and $u=0$, the model turns into a traditional type, in which the cooperators soon die out as represented in the blue area. With the increment of $\alpha$ and $u$, the colour from blue to green means that the density of cooperation is around 0.5 . In the plane, we can see that the combination mechanism impacts the density of cooperation, and most of the planes are yellow colour, which represents a high density of cooperators.

As shown in Figure 12, the snapshot of cooperators and defectors for different values of the parameters $u$ and $\alpha$ and the spatial evolution of strategies under three scenarios are depicted. As for the traditional type, the cooperator is soon invaded by a defector. All firms have chosen a strategy of defection. However, the introduction of heterogeneity of environment and environmental preference can lead to a different result, and defector initially invades cooperator; most firms have chosen a defection strategy. As the evolutionary process progresses, due to the environmental heterogeneity and preference, the firms gradually choose the cooperation strategy, but there are also defectors in this game eventually. With the larger values of $u$ and $\alpha$, the cooperative cluster rapidly expands in the system, occupies almost the entire system, and the level of cooperation remains stable. Compared with the previous two scenarios, it can promote more firms to choose cooperation strategy.

It is interesting to explore the evolution of cooperation under the environmental heterogeneity and preference for different steps, and the result is displayed in Figure 13. As for the traditional type, some firms initially formed the compact cluster, and then all firms choose to defect. Considering the coexistence of environmental and heterogeneity and preference, the compact clusters of cooperators are formed quickly, making defectors not invaded. The clusters change continuously over time, and the defectors also exist in the lattice network eventually. With the larger values of $u$ and $\alpha$, the situation becomes harmonious, many firms are attracted by the payoff of cooperation, and cooperators win the evolutionary race.

\section{Discussion}

From the above simulation results, we propose the following propositions.

Proposition 1. There is a threshold level of heterogeneity of the environment in which the firms choose to cooperate or defect both PDG and SDG.

In our study, we first investigate the correlation between the environment's heterogeneity and the density of cooperation. The heterogeneity of the environment signifies the contribution of the environment to fitness. The firm's cooperation has increased both PDG and SDG with the increased heterogeneity of the environment. Moreover, there is also a threshold level of heterogeneity of the environment in which firms' density presents three significant types. Suppose the environment's heterogeneity is more considerable than a threshold, and the increase of $u$ leads to complete cooperation. Otherwise, the density of cooperation maintains a mix-strategies or total defection value. For example, when $\alpha=2$ and $u>0.8$, all firms will choose to 


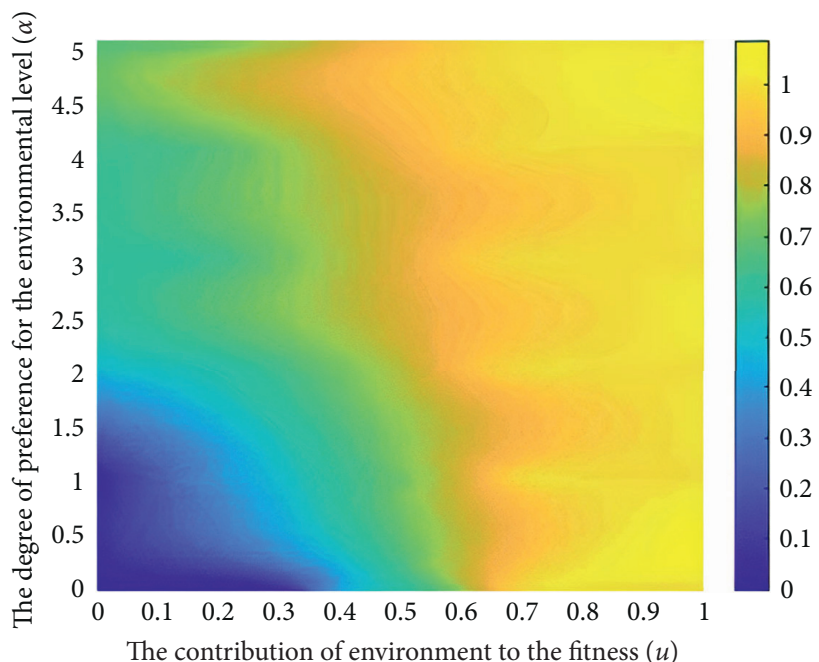

(a)

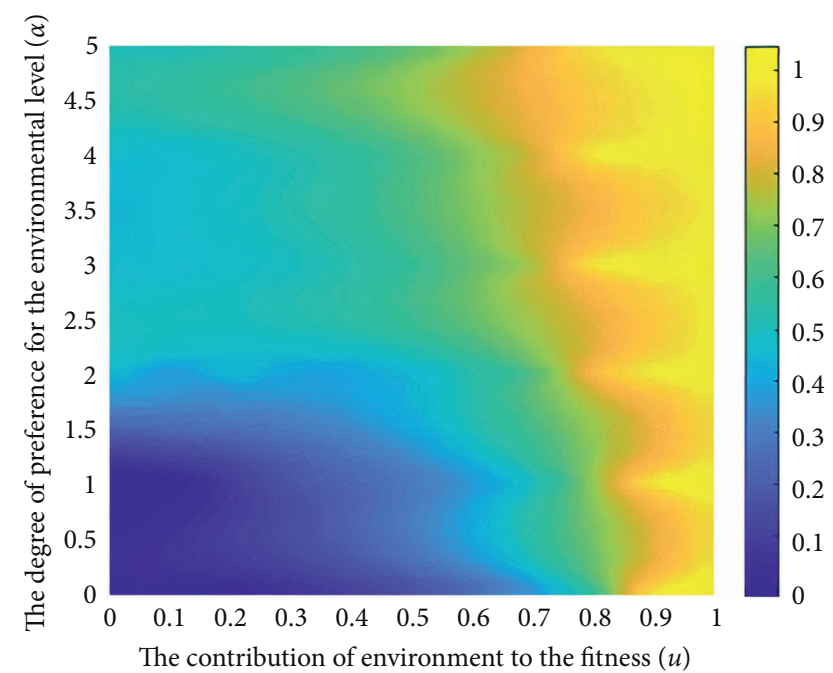

(b)

Figure 11: The colour-coded (see the bar on the right) density of cooperation $\rho_{c}$ on the $u-\alpha$ parameter plane. The depicted are obtained in $b=1.3$ ((a) for the PDG) and $r=0.8$ ((b) for the SDG). The yellow shading corresponds to the higher stationary density of cooperators.
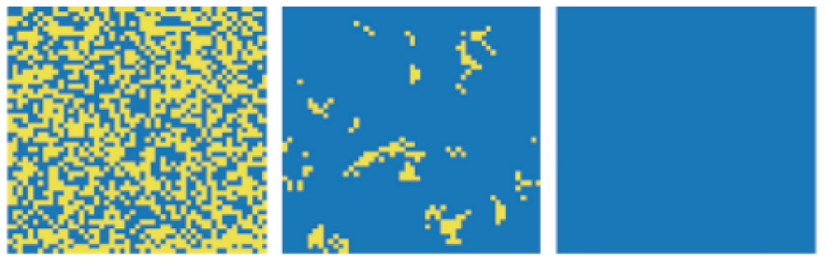

(a)
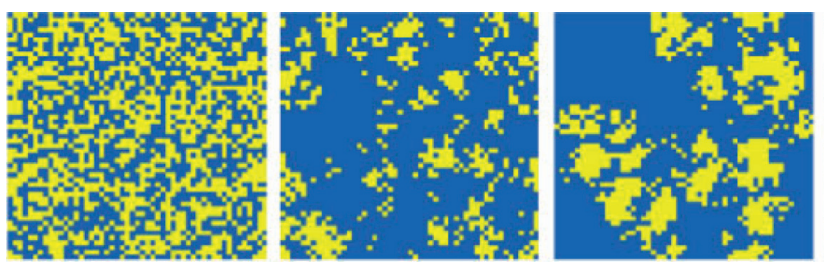

(b)
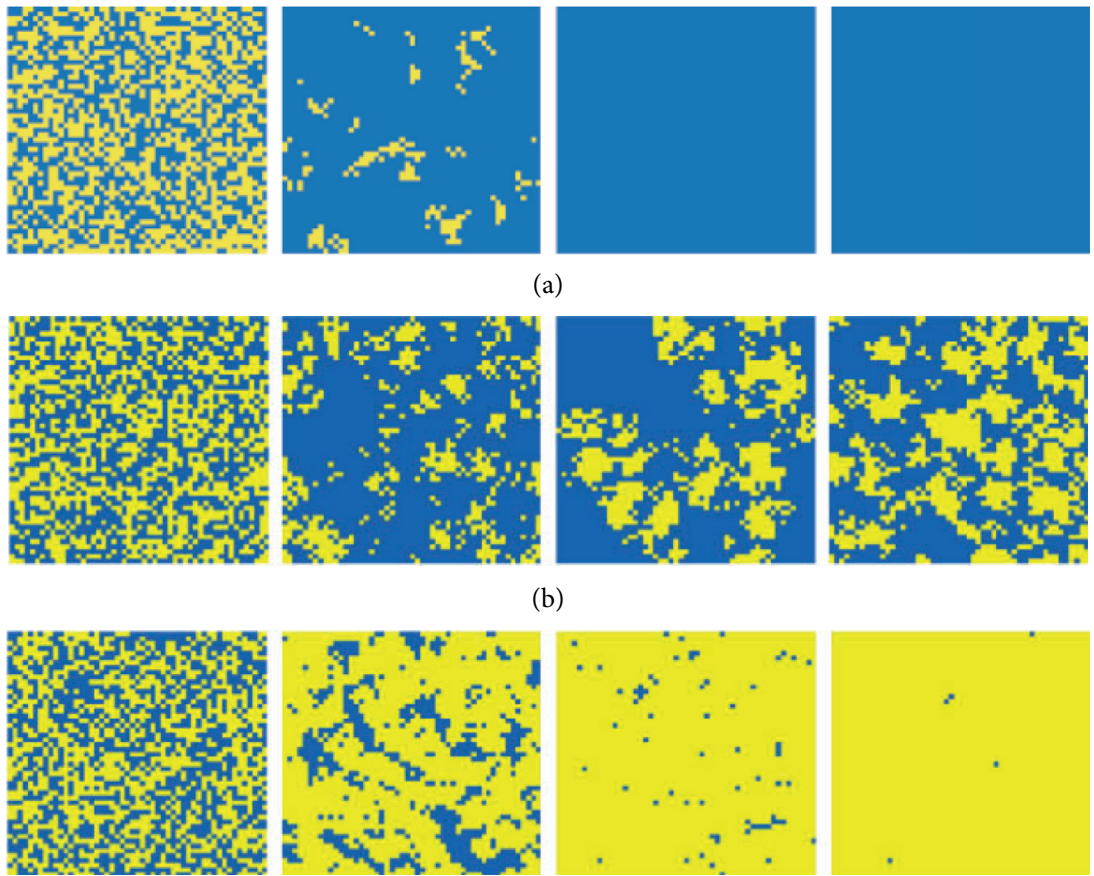

(c)

Figure 12: Characteristic snapshot of cooperators (yellow) and defectors (blue) for different values of the parameters $u$ and $\alpha$. (a) The traditional type $(u=0$ and $\alpha=0)$, (b) the coexistence of environmental heterogeneity and preference $(u=0.2$ and $\alpha=1)$, and (c) the more considerable heterogeneity of environment and environmental preference $(u=0.7$ and $\alpha=4)$. From left to right, the simulation steps are 0,5 , 50 , and 500. The depicted are obtained for $b=1.3$.

cooperate eventually regardless of the value of $b$ in PDG (Figure 5) and the value of $r$ in SDG (Figure 6).

Proposition 2. As the degree of preference for environmental level increases, the density of cooperating firms gradually increases in PDG but immediately stabilizes at a specific value in SDG.
The firm's fitness is also affected by the nearest neighbour's heterogeneity of the environment. The focal firm prefers to adopt the strategy, which has a higher level of the environment. As a result, we find that environmental preference improves the density of cooperation in PDG. Moreover, the more considerable degree of environment preference, the more significant density of cooperation 

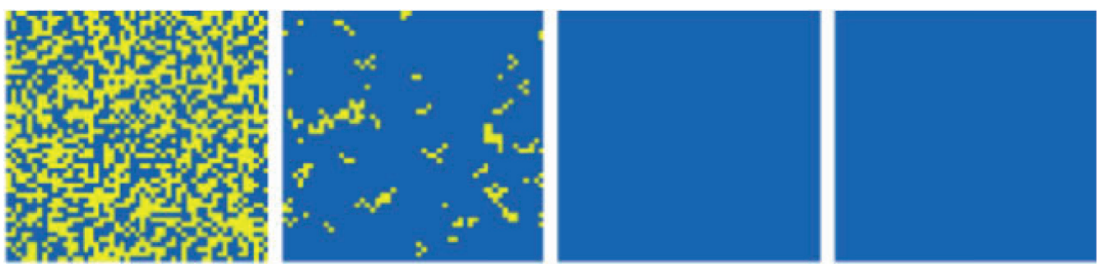

(a)
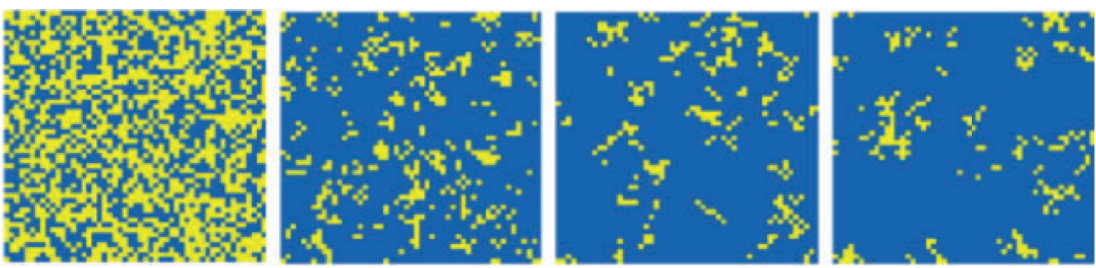

(b)
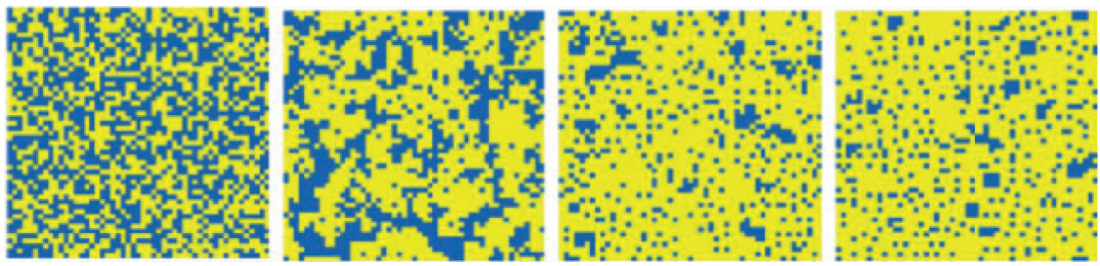

(c)

Figure 13: Characteristic snapshot of cooperators (yellow) and defectors (blue) for different values of the parameters $u$ and $\alpha$. (a) The traditional type $(u=0$ and $\alpha=0)$, (b) the coexistence of environmental heterogeneity and preference $(u=0.2$ and $\alpha=1)$, and (c) the larger heterogeneity of environment and environmental preference $(u=0.7$ and $\alpha=4)$. From left to right, the simulation steps are $0,5,50$, and 500 . The depicted are obtained for $r=0.8$.

(Figure 7). For example, when $\alpha=5$, environmental preference increases the density of cooperation from 0 to 0.7 . Compared to PDG, environmental preference has a different influence on the density of cooperation in SDG. With the increment of $\alpha$, the density of cooperation will quickly reach the maximum value. The more considerable value of the parameter $\alpha$, the less time it will take to attain a specific value (Figure 8). The specific value is reached in 400-time steps when $\alpha=2$. However, it is reached in 150 -time steps when $\alpha=4$.

Proposition 3. Under the coexistence heterogeneity and preference of environment, the promotion of cooperating firms' density is more obvious both PDG and SDG, and the heterogeneity of environment has more significant implications than an environmental preference on the density of cooperation.

With the increase of the degree of the heterogeneity of environment and the degree of preference for environmental level, the colour of the density of cooperation from blue to yellow which the area of cooperation density reaches the maximum (Figure 11). With the tremendous intensity of environmental heterogeneity and preference, a higher number of firms choose to cooperate.

Proposition 4. In the heterogeneity and preference of environment, the compact clusters of cooperative firms are formed to resist the invasion of defection, both PDG and SDG.
The numbers of cooperation and defection change over time, and we can see that the firms immediately formed cluster to resist defector invasion. In the practical, individual decisions and actions constitute the dynamics of groups, functions, organizations, and ultimately supply chains. Alliances are often formed to meet the challenges of the market and social forces. Effective and complete integration from suppliers to end-users will help firms improve operational performance and gain greater competitiveness. From Figures 12 and 13, the firms formed compact clusters with the same strategy, which make defectors are not invaded.

\section{Conclusions}

This paper presents the supply network evolution model based on game theory and CA to understand the evolution of $\mathrm{SN}$. They are capturing complex interdependencies, also known as reciprocal interdependencies. The simulation experiment results show the interconnected relationship behaviours of firms in the supply network. We have investigated the influence of environmental heterogeneity and preference on the evolution of cooperation in the supply network. The heterogeneity of the environment in which firms are located usually depends on their neighbours' average level.

Moreover, we further investigate the implication of environmental preference on the density of cooperation. If the firms' neighbours have a higher level of environment, the higher probability adopts the strategy which has a higher level of the environment. The two types of games applied in the 
experiments include PDG and SDG. We extend the heterogeneity of environment and environmental preference to the evolutionary game to explore cooperative behaviours in supply networks. The simulations are conducted on the CA model with eight neighbours. Simulations represent that when the heterogeneous environment and environmental preference are introduced, cooperation is promoted in the PDG and SDG.

The research found a positive correlation between the heterogeneous environment and cooperating firms' density both PDG and SDG. We further found that the environmental preference positively influences the density of cooperation regardless of PDG and SDG, but the density of cooperating firms gradually increases in PDG and immediately stabilizes at a specific value in SDG. Moreover, environmental preference has more influence on the density of cooperation than environmental heterogeneity. With the characteristic snapshot of cooperators and defectors for different values of the environmental heterogeneity and the environmental preference, it is found that firms that take into account the surrounding environment tend to cooperate and form the compact cluster.

The implication for management of this is that decisionmakers should consider the behaviours of their firm and investigate the strategies of their suppliers and the complex relationships between focal firms and suppliers. As time goes on, decision-makers should re-evaluate the relationship, payoff, and fitness when there are shifts in suppliers' strategies. To improve the level of cooperation, environmental heterogeneity and environmental preference have been applied. It is wise to consider the level of the environment of suppliers and use the environmental preference to increase the density of cooperation in supply networks.

A limitation of this study is that although payoff, environmental heterogeneity, and preference have been considered, and the CA lattice's supply network did not further involve random networks, small-world networks, and scalefree networks. Besides, there are differences in the impact of environmental preference in PDG and SDG models. From this point of view, future studies are to consider the impact of the stag-hunt game, hawk dove game, or other types of games on the number of cooperating firms with environmental preference. Secondly, it would be interesting to consider more firms' strategies and modify firms' strategies during simulation based on the evolving environmental heterogeneity and preference.

\section{Data Availability}

The data used to support the findings of this study are available from the corresponding author upon request.

\section{Conflicts of Interest}

The authors declare that they have no conflicts of interest.

\section{Acknowledgments}

This research was funded by the National Natural Science Foundation of China (no. 71473013) and the Fundamental
Research Funds for the Central Universities (no. 2019YJS072).

\section{References}

[1] H. Xia, "Improve the resilience of multilayer supply chain networks," Complexity, vol. 20209 pages, 2020.

[2] M. Sofitra, K. Takahashi, K. Morikawa, and K. Morikawa, “The coevolution of interconnected relationship strategies in supply networks," International Journal of Production Research, vol. 53, no. 22, pp. 6919-6936, 2015.

[3] S. Perera, M. Bell, and M. Bliemer, "Network science approach to modelling emergence and topological robustness of supply networks: a review and perspective," 2018, https://arxiv.org/ abs/1803.09913.

[4] M. R. Khaji and R. Shafaei, "A system dynamics approach for strategic partnering in supply networks," International Journal of Computer Integrated Manufacturing, vol. 24, no. 2, pp. 106-125, 2011.

[5] A. Brintrup, Y. Wang, and A. Tiwari, "Supply networks as complex systems: a network-science-based characterization," IEEE Systems Journal, vol. 11, no. 4, pp. 2170-2181, 2017.

[6] Y. Kim, T. Y. Choi, T. Yan, and K. Dooley, "Structural investigation of supply networks: a social network analysis approach," Journal of Operations Management, vol. 29, no. 3, pp. 194-211, 2011.

[7] A. Nair, C. Blome, T. Y. Choi, and G. Lee, "Re-visiting collaborative behavior in supply networks-structural embeddedness and the influence of contextual changes and sanctions," Journal of Purchasing and Supply Management, vol. 24, no. 2, pp. 135-150, 2018.

[8] G. Yang, C. Zhu, and W. Zhang, "Adaptive and probabilistic strategy evolution in dynamical networks," Physica A: Statistical Mechanics and its Applications, vol. 518, pp. 99-110, 2019.

[9] S. Perera, D. Kasthurirathna, M. Bell, and M. Bliemer, "Topological rationality of supply chain networks," International Journal of Production Research, vol. 58, pp. 1-24, 2019.

[10] N. Rashedi and H. Kebriaei, "Cooperative and non-cooperative Nash solution for linear supply function equilibrium game," Applied Mathematics and Computation, vol. 244, pp. 794-808, 2014.

[11] H. Barcelo and V. Capraro, "Group size effect on cooperation in one-shot social dilemmas," Scientific Reports, vol. 5, 2015.

[12] J. Zhao, C. Luo, and Y. Zheng, "Evolutionary dynamics of the cooperation clusters on interdependent networks," Physica A: Statistical Mechanics and its Applications, vol. 517, pp. 132140, 2019.

[13] L. John, P. McCormick, T. McCormick, G. R. McNeill, and J. Boardman, "Working to understand cooperative forces in government extended enterprises: concepts and methodology,” IEEE Systems Journal, vol. 6, no. 4, pp. 675-687, 2012.

[14] F. Dercole, F. Della Rossa, and C. Piccardi, "Direct reciprocity and model-predictive rationality explain network reciprocity over social ties," Scientific Reports, vol. 9, 2019.

[15] C. Liu, J. Shi, T. Li, and J. Liu, "Aspiration driven coevolution resolves social dilemmas in networks," Applied Mathematics and Computation, vol. 342, pp. 247-254, 2019.

[16] M. Wiedermann, J. F. Donges, J. Heitzig et al., "Macroscopic description of complex adaptive networks coevolving with dynamic node states," Physical Review E, Statistical, Nonlinear, and Soft Matter Physics, vol. 91, p. 52801, 2015.

[17] H. P. Thadakamalla, U. N. Raghavan, S. Kumara, and A. Albert, "Survivability of multiagent-based supply networks: 
a topological perspective," IEEE Intelligent Systems, vol. 19, no. 5, pp. 24-31, 2004.

[18] J. Liu, H. Meng, W. Wang, Z. Xie, and Q. Yu, "Evolution of cooperation on independent networks: the influence of asymmetric information sharing updating mechanism," Applied Mathematics and Computation, vol. 340, pp. 234-241, 2019.

[19] T. Sakiyama and I. Arizono, "An adaptive replacement of the rule update triggers the cooperative evolution in the HawkDove game," Chaos, Solitons \& Fractals, vol. 121, pp. 59-62, 2019.

[20] C. Xia, J. Wang, L. Wang, S. Sun, J. Sun, and J. Wang, "Role of update dynamics in the collective cooperation on the spatial snowdrift games: beyond unconditional imitation and replicator dynamics," Chaos, Solitons \& Fractals, vol. 45, no. 9-10, pp. 1239-1245, 2012.

[21] G.-H. Cui, Z. Wang, Y.-C. Yang, S.-W. Tian, and J. Yue, "Heterogeneous game resource distributions promote cooperation in spatial prisoner's dilemma game," Physica A: Statistical Mechanics and its Applications, vol. 490, pp. 11911200, 2018.

[22] K. Huang, X. Zheng, and Y. Su, "Effect of heterogeneous subpopulations on the evolution of cooperation," Applied Mathematics and Computation, vol. 270, pp. 681-687, 2015.

[23] J. Jin, C. Chu, C. Shen et al., "Heterogeneous fitness promotes cooperation in the spatial prisoner's dilemma game," Chaos, Solitons \& Fractals, vol. 106, pp. 141-146, 2018.

[24] L. Sun, S. Rajiv, and J. Chu, "Beyond the more the merrier: the variety effect and consumer heterogeneity in system markets," International Journal of Research in Marketing, vol. 33, no. 2, pp. 261-275, 2016.

[25] G. Li, Y.-G. Gu, and Z.-H. Song, "Evolution of cooperation on heterogeneous supply networks," International Journal of Production Research, vol. 51, no. 13, pp. 3894-3902, 2013.

[26] Y. E. Wu, Z. Zhang, and S. Chang, "Heterogeneous indirect reciprocity promotes the evolution of cooperation in structured populations," Chaos: An Interdisciplinary Journal of Nonlinear Science, vol. 28, no. 12, p. 123108, 2018.

[27] C. Colon and M. Ghil, "Economic networks: heterogeneityinduced vulnerability and loss of synchronization," Chaos: An Interdisciplinary Journal of Nonlinear Science, vol. 27, no. 12, p. 126703, 2017.

[28] B. Andres and R. Poler, "A decision support system for the collaborative selection of strategies in enterprise networks," Decision Support Systems, vol. 91, pp. 113-123, 2016.

[29] N. K. Verma and A. K. Chatterjee, "A multiple-retailer replenishment model under VMI: accounting for the retailer heterogeneity," Computers \& Industrial Engineering, vol. 104, pp. 175-187, 2017.

[30] M. De Keizer, R. Akkerman, M. Grunow, J. M. Bloemhof, R. Haijema, and J. G. A. J. Van Der Vorst, "Logistics network design for perishable products with heterogeneous quality decay," European Journal of Operational Research, vol. 262, no. 2, pp. 535-549, 2017.

[31] A. Nakkas and Y. Xu, "The impact of valuation heterogeneity on equilibrium prices in supply chain networks," Production and Operations Management, vol. 28, no. 2, pp. 241-257, 2019.

[32] W. Zhou, T. Jing, W. Cheng, T. Chen, and Y. Huo, "Combinatorial auction based spectrum allocation under heterogeneous supply and demand," Computer Communications, vol. 60, pp. 109-118, 2015.

[33] H. Liu, N. Yang, Z. Yang, J. Lin, and Y. Zhang, “The impact of firm heterogeneity and awareness in modeling risk propagation on multiplex networks," Physica A: Statistical Mechanics and its Applications, vol. 539, p. 122919, 2020.

[34] A. Verhetsel, R. Kessels, P. Goos, T. Zijlstra, N. Blomme, and J. Cant, "Location of logistics companies: a stated preference study to disentangle the impact of accessibility," Journal of Transport Geography, vol. 42, pp. 110-121, 2015.

[35] S. Chang, Z. Zhang, Y. Li, Y. E. Wu, and Y. Xie, "Investment preference promotes cooperation in spatial public goods game," PLoS One, vol. 13, Article ID e206486, 2018.

[36] S. Zhang, Z. Zhang, Y. E. Wu, M. Yan, and Y. Li, "Strategy preference promotes cooperation in spatial evolutionary games," Physica A: Statistical Mechanics and its Applications, vol. 514, pp. 181-188, 2019.

[37] Y. Li and H. Ye, "Effect of the migration mechanism based on risk preference on the evolution of cooperation," Applied Mathematics and Computation, vol. 320, pp. 621-632, 2018.

[38] G. Yu, F. Li, and Y. Yang, "Robust supply chain networks design and ambiguous risk preferences," International Journal of Production Research, vol. 55, no. 4, pp. 1168-1182, 2017.

[39] C. Du and J. Li, "Preferential learning and memory resolve social dilemma," Chaos, Solitons \& Fractals, vol. 110, pp. 16-19, 2018.

[40] C. Huang, Q. Dai, H. Cheng, and H. Li, "Preferential selection based on degree difference in the spatial prisoner's dilemma games," EPL (Europhysics Letters), vol. 120, no. 1, p. 18001, 2017.

[41] M. C. Cooper and L. M. Ellram, "Characteristics of supply chain management and the implications for purchasing and logistics strategy," The International Journal of Logistics Management, vol. 4, no. 2, pp. 13-24, 1993.

[42] J. Rodewald, J. Colombi, K. Oyama, and A. Johnson, "Methodology for simulation and analysis of complex adaptive supply network structure and dynamics using information theory," Entropy, vol. 18, no. 10, p. 367, 2016.

[43] S. S. Perera, M. G. H. Bell, M. Piraveenan, D. Kasthurirathna, and M. Parhi, "Topological structure of manufacturing industry supply chain networks," Complexity, vol. 2018, 23 pages, 2018.

[44] Y. S. H. R. Jianbang Du, "The relationship of delivery frequency with the cost and resource operational efficiency: a case study of Jingdong logistics," Mathematics and Computer Science, vol. 3, pp. 129-140, 2018.

[45] T. Y. Choi and D. R. Krause, "The supply base and its complexity: implications for transaction costs, risks, responsiveness, and innovation," Journal of Operations Management, vol. 24, no. 5, pp. 637-652, 2006.

[46] E. J. S. Hearnshaw and M. M. J. Wilson, "A complex network approach to supply chain network theory," International Journal of Operations \& Production Management, vol. 33, no. 4, pp. 442-469, 2013.

[47] M. A. Bellamy and R. C. Basole, "Network analysis of supply chain systems: a systematic review and future research," Systems Engineering, vol. 16, no. 2, pp. 235-249, 2013.

[48] A. Nair, R. Narasimhan, and T. Y. Choi, "Supply networks as a complex adaptive system: toward simulation-based theory building on evolutionary decision making," Decision Sciences, vol. 40, no. 4, pp. 783-815, 2009.

[49] Y. E. Wu, Z. Zhang, and S. Chang, "Reciprocal reward promotes the evolution of cooperation in structured populations," Chaos, Solitons \& Fractals, vol. 119, pp. 230-236, 2019.

[50] J. Jin, C. Shen, C. Chu, and L. Shi, "Incorporating dominant environment into individual fitness promotes cooperation in 
the spatial prisoners' dilemma game," Chaos, Solitons \& Fractals, vol. 96, pp. 70-75, 2017.

[51] J. Xu, Z. Deng, B. Gao et al., "Popularity-driven strategy updating rule promotes cooperation in the spatial prisoner's dilemma game," Applied Mathematics and Computation, vol. 353, pp. 82-87, 2019.

[52] M. A. Nowak and R. M. May, "Evolutionary games and spatial chaos," Nature, vol. 359, no. 6398, pp. 826-829, 1992.

[53] G. Szabó and G. Fáth, "Evolutionary games on graphs," Physics Reports, vol. 446, no. 4-6, pp. 97-216, 2007.

[54] H. Guo, C. Shen, D. Dai, M. Zhang, C. Chu, and L. Shi, "Environment promotes the evolution of cooperation in spatial voluntary prisoner's dilemma game," Applied Mathematics and Computation, vol. 315, pp. 47-53, 2017.

[55] Y. E. Wu, S. Zhang, and Z. Zhang, "Environment-based preference selection promotes cooperation in spatial prisoner's dilemma game," Scientific Reports, vol. 8, 2018.

[56] P. Zhu, X. Wang, D. Jia, Y. Guo, S. Li, and C. Chu, "Investigating the co-evolution of node reputation and edge-strategy in prisoner's dilemma game," Applied Mathematics and Computation, vol. 386, p. 125474, 2020.

[57] P. Zhu, Z. Song, H. Guo, Z. Wang, and T. Zhao, "Adaptive willingness resolves social dilemma in network populations," Chaos: An Interdisciplinary Journal of Nonlinear Science, vol. 29, no. 11, p. 113114, 2019. 